\title{
Mean P Wave Height
}

National Cancer Institute

\section{Source}

National Cancer Institute. Mean P Wave Height. NCI Thesaurus. Code C62124.

The average (mean) height (usually measured in $\mathrm{mm}$ ) of the maximum deflection from baseline of the $\mathrm{P}$ wave (representing atrial depolarization), obtained from a set of measurements of the P wave, from a single lead or set of leads. Typically this measurement is obtained by analysis of Lead II. 